\title{
Robust Extraction and Simplification of 2D Symmetric Tensor Field Topology
}

Jochen Jankowai, Bei Wang and Ingrid Hotz

The self-archived postprint version of this journal article is available at Linköping University Institutional Repository (DiVA):

http://urn.kb.se/resolve?urn=urn:nbn:se:liu:diva-160176

N.B.: When citing this work, cite the original publication.

Jankowai, J., Wang, B., Hotz, I., (2019), Robust Extraction and Simplification of 2D Symmetric Tensor Field Topology, Computer graphics forum (Print), 38(3), 337-349. https://doi.org/10.1111/cgf.13693

Original publication available at:

https://doi.org/10.1111/cgf.13693

Copyright: Wiley (12 months)

http://eu.wiley.com/WileyCDA/ 


\title{
Robust Extraction and Simplification of 2D Symmetric Tensor Field Topology
}

\author{
Jochen Jankowai $^{\dagger 1}$ (D), Bei Wang ${ }^{\ddagger 2}$ (D) , Ingrid Hotz ${ }^{\S 1}$ (iD \\ ${ }^{1}$ Scientific Visualization Group, Linköpings University, Sweden \\ ${ }^{2}$ Scientific Computing and Imaging Institute, University of Utah, USA
}

\begin{abstract}
In this work, we propose a controlled simplification strategy for degenerated points in symmetric $2 D$ tensor fields that is based on the topological notion of robustness. Robustness measures the structural stability of the degenerate points with respect to variation in the underlying field. We consider an entire pipeline for generating a hierarchical set of degenerate points based on their robustness values. Such a pipeline includes the following steps: the stable extraction and classification of degenerate points using an edge labeling algorithm, the computation and assignment of robustness values to the degenerate points, and the construction of a simplification hierarchy. We also discuss the challenges that arise from the discretization and interpolation of real world data.
\end{abstract}

CCS Concepts

- Human-centered computing $\rightarrow$ Scientific visualization; $\bullet$ Computing methodologies $\rightarrow$ Scientific visualization;

\section{Introduction}

Topology provides a powerful instrument for the structural analysis of complex data. A major breakthrough for research in topological data analysis is the introduction of stability measures based on the notion of persistence [ELZ02], that lead to hierarchical simplifications of data. However, most of the persistence-based simplification is restricted to the study of scalar fields. There is a large potential for the application of topological simplification for vector field and tensor field analysis.

For the analysis of 2D tensor fields, topology provides a segmentation of the domain into regions of similar behavior using tensor lines, i.e., lines following the eigenvector directions of tensors. The degenerate points are the topological features important in determining the irregularities within those segmentations [ASNZH11]. Such a structural analysis of tensor fields can be beneficial in a wide range of applications. For example, a direct application of topological segmentation is surface re-meshing [ACSD*03, MK04] guided by the curvature tensor field. In material science, directional properties of the tensor fields are interesting for the analysis of stress tensor fields. Kratz et al. have shown recently that tensor lines play an important role in the analysis of stress propagation in mate-

\footnotetext{
$\dagger$ e-mail: jochen.jankowai@liu.se

† e-mail: beiwang@sci.utah.edu

$\S$ e-mail: ingrid.hotz@liu.se
}

rial [KSZ*14]. In diffusion tensor imaging (DTI), tensor lines are related to neural fiber tracts in the brain.

Topology has not been used much in the field of tensor field analysis; however, together with the right simplification strategy, it can add a valuable new perspective. The most important requirement in applying tensor field topology to real world applications is the extraction of the topology representing the core structure of data, that is stable with respect to small changes in the field. In regions

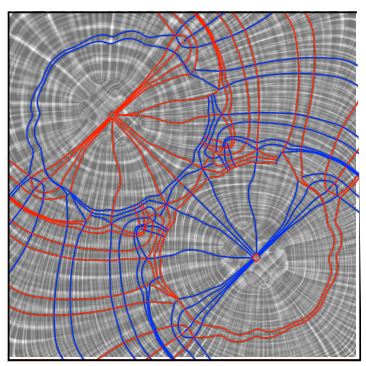

(a)

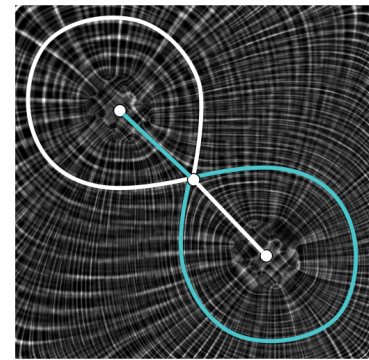

(b)
Figure 1: A topological skeleton of a $2 D$ slice from a stress tensor field. The background texture is aligned to the eigenvector fields. Left: the red and blue lines represent the full topological skeleton of the major and minor eigenvector fields. Right: the structure of the field that one would like to extract, here sketched by hand. 
of high isotropy, however, the topological skeleton of a tensor field typically becomes very complex, with many degenerate points. A large collection of the topological features is not stable under small changes of the field and therefore is less significant from a global perspective. The full topological skeleton of a slice of a stress tensor field is shown in Fig. 1(a). In comparison, Fig. 1(b) shows a hand sketch of a desirable, ideal topological summary. A scheme to simplify the topology by removing less stable and thus significant structures (the noise) while preserving the significant ones (the signal) is therefore necessary.

In this work, we focus on extracting and simplifying the topology of a 2D symmetric tensor field by assigning stability measures to degenerate points. Wang and Hotz [WH17] have introduced the theoretical notion of robustness for tensor fields, which measures the stability of degenerate points with respect to perturbations of the fields. We build upon this theoretical formulation and focus on algorithmic investigations, implementational details and its applications to real world datasets. In particular:

- We present a framework that generates a hierarchy among pairs of degenerate points based on their robustness. Such a hierarchy serves as the basis for a hierarchical simplification scheme where points with low robustness are candidates for simplification (cancellation).

- We discuss the entire algorithmic pipeline from the stable extraction of degenerate points to the computation of the hierarchy. The proposed algorithm is designed to be simple and consistent for data given on a triangulated domain. The entire pipeline does not require the computation of eigenvectors and eigenvalues and is purely based on the tensor components.

- We extract and classify degenerate cells using an edge-labeling approach, which restricts all numerical computations to a preprocessing step. To deal with degenerate cases, such as degenerate points on edges or vertices, we propose a symbolical perturbation of the field that consistently pushes the degenerate points into neighboring cells.

- For evaluation, we apply the robustness framework, for the first time, to real world data sets from mechanical engineering and DTI, discuss the interpretability of the results, and demonstrate the potential of such a framework for tensor field analysis.

\section{Related work}

Tensors as linear mappings between vectors play an important role in many applications ranging from stress tensors in mechanical engineering, to diffusion tensors in medicine, and curvature tensors in geometry. Accordingly, there has been much work dealing with tensor field visualization. An overview of the most important methods can be found in the state-of-the-art report by Kratz et a. [KASH13]. Here, we discuss the most relevant ones surrounding tensor field topology and topological simplification.

Tensor Field Topology. Since the introduction of tensor field topology to visualization by Delmarcelle [De194], some efforts have been made in simplifying the topology of tensor fields. Tricoche has contributed various approaches to simplify tensor fields in his $\mathrm{PhD}$ thesis [Tri02]; for instance, merging degenerate points for cancellation based upon their connectivity by separatrices [TSH01].
Tricoche et al. [TS03] have also proposed a method to merge clusters of degenerate points which either cancel or generate higher order degenerate points, using geometric criteria such as proximity. Thereby, the connection of degenerate points with a separatrix is not required. Their method can be generally applied with any importance measures. Zhang et al. [ZHT07] have proposed a system for tensor field design, which also includes a tensor field editing and simplification step. This system includes a user-guided smoothing procedure within a specified region that keeps the values fixed on the boundary. This procedure is not an explicit degenerate point merging but a repeated averaging process. Similar tensor field smoothing approaches have been applied by Alliez et al. [ACSD*03] and Marinov et al. [MK04] for surface remeshing. Nair et al. [SNAHH11] have introduced an implicit simplification via adopting the triangulation of the domain without changing the tensors given on the vertices. The emphasis in all this work is on the simplification procedure of the tensor field; however, no topological importance measure for a hierarchical cancellation of the degenerate points is introduced. In contrast to our work, the notion of stability with respect to perturbations of the fields has not been considered in any of these simplification strategies.

Robustness for Vector Fields. Robustness is closely related to the notion of persistence [ELZ02], which has successfully introduced a hierarchical structure of topological features for scalar fields. Motivated to find a similar hierarchical structure for vector fields, the concept of robustness has been introduced to rank vector field critical points by their stability. Robustness is based on the algebraic concept of well diagrams and well group theory [EMP10, EMP11, CPS12]. It quantifies the stability of critical points with respect to the minimum amount of perturbation required to remove them. For the analysis and visualization of vector fields, robustness has been applied to 2D and 3D fields [SWCR14, SWCR15, SRW*16]. A robustness-based vector field simplification strategy has been introduced independent of the topological skeleton [SWCR14]; for 3D vector fields, robustness gives rise to the first simplification method using critical point cancellation [SRW*16]. Recently, the concept of robustness has been extended to tensor field topology [WH17]. This theoretical framework establishes the basis for the computational approach presented in this paper.

Comparison with Previous Work. The work in [SWCR15] establishes the theoretical and algorithmic framework for robustnessbased simplification of steady and unsteady vector fields. While the simplification frameworks of both vector and tensor fields are based on the well group theory, the one for tensor field requires additional, nontrivial algorithmic development (such as the study of tensor field perturbation and anisotropy vector field mapping, see Section 3.2). In addition, there is practically no overlap in terms of implementation between the vector field and tensor field setting. While [WH17] explores robustness for tensor fields theoretically and analytically, we discuss nontrivial algorithmic aspects, including degenerate point extraction and robustness computation in the piecewise linear (PL) settings. In addition, we investigate real world applications. Our main contribution is the implementation and construction of hierarchical relations among degenerate points based on robustness to support simplification of the topology. 


\section{Background}

In this section, we briefly review some basics on tensor field topology and introduce the concept of robustness from topological data analysis. We keep the relevant technical details to a minimal and refer to [WH17] for an in-depth description of robustness for tensor field topology. We restrict our attention to the setting of symmetric tensor fields of second order, defined on a $2 \mathrm{D}$ domain $\mathbb{R}^{2}$; when talking about tensors for the rest of this paper, we always refer to 2D symmetric tensors of second order.

\subsection{Background in Tensor Field Topology}

In tensor field topology, the topological skeleton segments the domain in regions of uniform tensorline behavior. The skeleton consists of tensor lines connecting the degenerate points, where both eigenvalues are the same [De194]. We review relevant concepts such as symmetric tensor field, eigenvectors, degenerate points, separatrices, and space of deviators.

2D Symmetric Second-Order Tensor Fields. In the context of this paper, a tensor $T$ is a linear operator that associates any vector $v$ with another vector $u=T \cdot v$, where $v$ and $u$ are vectors in $\mathbb{R}^{2}$. Given a basis of $\mathbb{R}^{2}$, a tensor $T$ can be represented by a symmetric $2 \times 2$ matrix, $T=\left[\begin{array}{ll}t_{11} & t_{12} \\ t_{12} & t_{22}\end{array}\right]$. A symmetric tensor field $\mathbb{T}$ is a mapping that assigns to each position $x=\left(x_{1}, x_{2}\right) \in \mathbb{R}^{2}$ a symmetric tensor, that is, $\mathbb{T}: \mathbb{R}^{2} \rightarrow \mathscr{T}^{2}$, where $\mathscr{T}^{2}$ denotes the set of all second-order symmetric tensors.

At a fixed location $x \in \mathbb{R}^{2}$, set $\mathbb{T}(x)=T$. The eigenvectors $\mathbf{e}_{i} \in \mathbb{R}^{2}$ with associated eigenvalues $\lambda_{i}$ of $T$ are defined by the eigenvector equation $T \mathbf{e}_{i}=\lambda_{i} \mathbf{e}_{i}$ (for $i \in\{1,2\}$ ) and $\mathbf{e}_{i} \neq 0$. We impose an ordering of the eigenvectors such that $\lambda_{1} \geq \lambda_{2}$. We refer to the larger and smaller eigenvalues as the major and minor eigenvalues, respectively; their respective eigenvectors are called the major and minor eigenvectors. We then define the two eigenvector fields, referred to as the major and minor eigenvector fields, respectively. These eigenvector fields are not continuous at the degenerate points defined below.

Degenerate Points and Separatrices. Degenerate points $x_{0} \in \mathbb{R}^{2}$ are defined as points where the eigenvalues are identical $\lambda_{1}\left(x_{0}\right)=$ $\lambda_{2}\left(x_{0}\right)$. In this case the isotropic tensor $T$ is proportional to the unit tensor, and its eigenvector directions are not uniquely defined. Such points play a similar role for tensor field topology as do zeros for vector field topology.

Isolated degenerate points can be classified by their winding number, which counts the number of rotations of the eigenvector field when moving along a simple curve enclosing the degenerate point. This number is an integer multiple of $1 / 2$. The winding number does not depend on the curve as long as it does not include another degenerate point. Linear fields have two different kinds of degenerate points: trisectors with winding number $-1 / 2$ and wedge points with winding number $+1 / 2$, as shown in Fig. 2 . The occurrence of half-integer-valued winding numbers is the most fundamental difference between vector fields and eigenvector fields, because eigenvectors have no orientations. According to the index theory for functions, the degree (or index) of a degenerate point is twice its winding number. For a path-connected component $C \subseteq \mathbb{R}^{2}$ containing a set of degenerate points $\left\{x_{1}, x_{2}, \ldots, x_{n}\right\}$, the degree $\mathrm{d} e g(C)$ of the tensor field restricted to the boundary $\partial C$ is the sum of the degrees at the poionts $x_{i}$.

Separatrices are tensorlines (i.e., lines tangential to the eigenvector field) connecting the degenerate points. Three separatrices are always attached to a trisector. Wedge points always have at least one separatrix attached; however, the number of attaching separatrices is not limited.

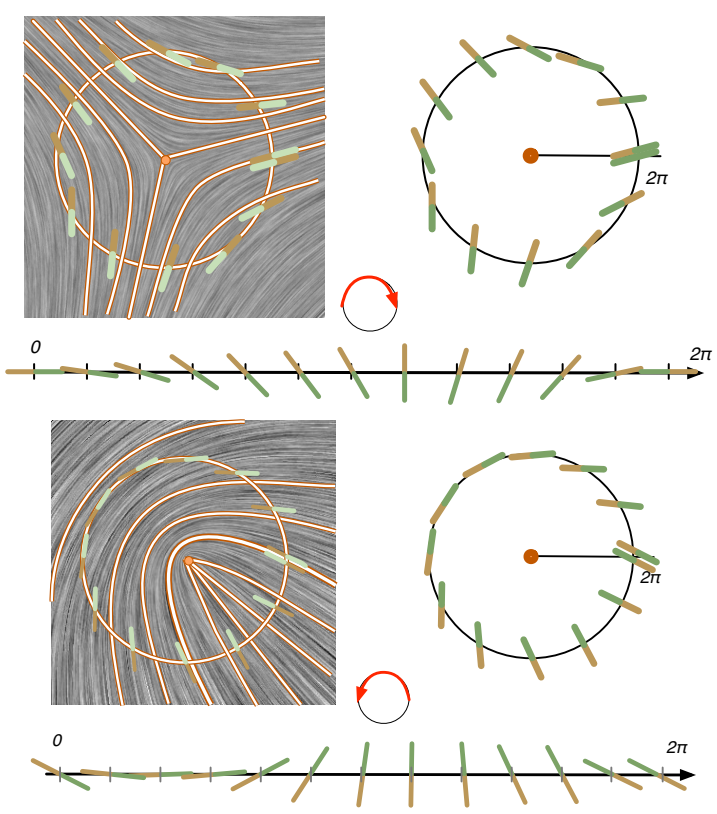

Figure 2: Typical structure of the eigenvector field in the vicinity of degenerate points for linear tensor fields. The winding number of a degenerate point counts the number of rotations when circling once around the degenerate point. In the linear case, degenerate points have a winding number of $\pm 1 / 2$. Top: a trisector with winding number $-1 / 2$. Bottom: a wedge point with winding number $+1 / 2$. Space of Deviators. The space of deviators $\mathscr{D}^{2}$ is a subspace of the space of symmetric tensors $\mathscr{T}^{2}$ containing only traceless tensors. The deviator $D$ is the projection $p$ of a tensor $T$ onto the space of deviators. It can be interpreted as the anisotropic part of the tensor and is defined as

$$
D:=p(T)=T-\frac{\operatorname{tr}(T)}{2} I,
$$

where $I$ represents the unit tensor and $\operatorname{tr}(T)$ is the trace of $T$. We use the following notation for its matrix representation:

$$
D=\left[\begin{array}{cc}
\Delta & F \\
F & -\Delta
\end{array}\right]
$$

If the tensor $T$ is degenerate, then $D$ is also degenerate. The eigenvectors of $D$ coincide with the eigenvectors of $T$. The topological structure of the deviator field $\mathbb{D}: \mathbb{R}^{2} \rightarrow \mathscr{D}^{2}$ (formed by $D$ ) is the same as that of the original field $\mathbb{T}: \mathbb{R}^{2} \rightarrow \mathscr{T}^{2}$ (formed by $T$ ). The structure of $\mathbb{D}$ will serve as the basis for the topological analysis of the original tensor field. 


\subsection{Robustness for Tensor Fields}

In this section, we review the work in [WH17], which establishes the theoretical formulations of robustness for tensor field topology. The robustness quantifies the stability of degenerate points with respect to the minimum amount of perturbation in the tensor fields required to remove them. Although the robustness for vector field topology [CPS12, SRW*16, SW14, SWCR15, WRS*13] is a well-founded framework based upon the well group theory [EMP10, EMP11], when transferring the theory of robustness for vector fields to tensor fields, the essential questions are: (i) how should perturbations of tensor fields be defined and how can they be interpreted as easily tractable properties of the fields; and (ii) how can we relate these properties to our features of interest, that is, the degenerate points.

In the following section, we first define the $r$-perturbation for tensor fields, and then we introduce an isometric mapping to an anisotropy vector field, which establishes a correlation between the perturbation and the stability of degenerate points. The isometry of the space of deviators equipped with the Frobenius norm and the space of vectors equipped with the $L_{2}$ norm give access to the framework developed for measuring the robustness of vector fields [CPS12, WRS*13].

Tensor Field Perturbation. The distance between two tensor fields is defined based on the Frobenius norm for tensors. For a tensor $T \in$ $\mathscr{T}^{2}$ given in its matrix form, its Frobenius norm $\|T\|_{F}$ is defined such that $\|T\|_{F}^{2}=\sum_{i=1}^{2} \sum_{i=j}^{2} t_{i j}^{2}=\lambda_{1}^{2}+\lambda_{2}^{2}$. For the deviator part $D$ of $T,\|D\|_{F}^{2}=\frac{1}{2}\left(\lambda_{1}-\lambda_{2}\right)^{2}$, where $\lambda_{1}$ and $\lambda_{2}$ are the eigenvalues of $T$. This corresponds to an anisotropy measure typically used for failure analysis of stress tensors. The Frobenius norm induces a metric on $\mathscr{T}^{2}$, that is, for $T, T^{\prime} \in \mathscr{T}^{2}: d_{F}\left(T, T^{\prime}\right)=\left\|T-T^{\prime}\right\|_{F}$. Let $D$ and $D^{\prime}$ be their corresponding deviator parts. Since computing the deviator of a tensor represents a projection on the subspace $\mathscr{D}^{2}$, we have $d_{F}\left(D, D^{\prime}\right) \leq d_{F}\left(T, T^{\prime}\right)$.

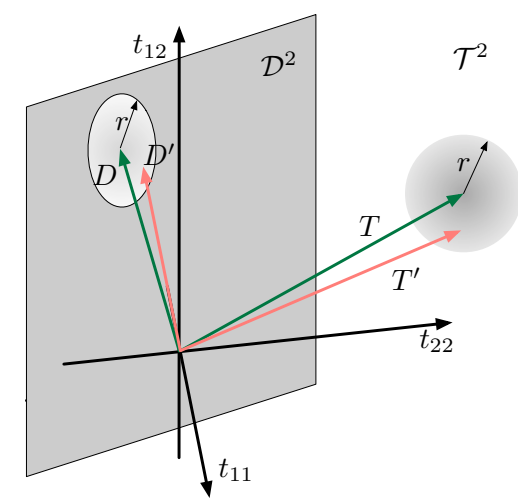

Figure 3: Geometric interpretation of an r-perturbation of a tensor field at a point $x$ in the space of tensors. The gray plane represents the deviatoric space $\mathscr{D}^{2}$ with $\operatorname{tr}(T)=0, \forall T \in \mathscr{T}^{2}$. If a tensor $T^{\prime}$ at $x$ is a r-perturbation of a tensor $T \in \mathscr{T}^{2}$, that is, $T^{\prime}$ falls within a ball of radius $r$ centered at $T$ in $\mathscr{T}^{2}$, then its deviator $D^{\prime}$ is a $r$-perturbation of $D \in \mathscr{D}^{2}$, that is, $D^{\prime}$ falls within a ball of radius $r$ centered at $D$ in $\mathscr{D}^{2}$.
Let $\mathbb{T}, \mathbb{T}^{\prime}: \mathbb{R}^{2} \rightarrow \mathscr{T}^{2}$ be two continuous $2 \mathrm{D}$ tensor fields. Their distance is defined as $d\left(\mathbb{T}, \mathbb{T}^{\prime}\right)=\sup _{x \in \mathbb{R}^{2}}\left\|\mathbb{T}(x)-\mathbb{T}^{\prime}(x)\right\|_{F}$. An $r$ perturbation of $\mathbb{T}$ is defined as a mapping $\mathbb{T}^{\prime}$ such that $d\left(\mathbb{T}, \mathbb{T}^{\prime}\right) \leq r$. This means that for each point $x \in \mathbb{R}^{2}$, the tensor $T^{\prime}=\mathbb{T}^{\prime}(x)$ lies within a sphere of radius $r$ in $\mathscr{T}^{2}$ centered at the tensor $T=\mathbb{T}(x)$, as shown in Fig. 3. Now let $\mathbb{D}, \mathbb{D}^{\prime}$ be their corresponding deviator fields. Since $d_{F}\left(D, D^{\prime}\right) \leq d_{F}\left(T, T^{\prime}\right)$, and that the tensor field $\mathbb{T}^{\prime}$ is an $r$-perturbation of the tensor field $\mathbb{T}$ (i.e. $d_{F}\left(T, T^{\prime}\right)<r$ ); it follows that the deviator field $\mathbb{D}^{\prime}$ is also an $r$-perturbation of $\mathbb{D}$.

Anisotropy Vector Fields. We now review the notion of an anisotropy vector field. The anisotropy vector field $\mathbb{A}: \mathbb{R}^{2} \rightarrow \mathbb{R}^{2}$ is defined by combining the projection $p: \mathscr{T}^{2} \rightarrow \mathscr{D}^{2}$ with an isometric mapping $\Omega: \mathscr{D}^{2} \rightarrow \mathbb{R}^{2}$ (using the Frobenius norm for tensors and the $L_{2}$ norm for vectors) [WH17], as shown in Fig. 4. Via such a mapping, the degenerate points of the tensor fields are mapped to the zeros in the anisotropy vector field, which allows us to treat $2 \mathrm{D}$ tensor fields in an analogous way as $2 \mathrm{D}$ vector fields.

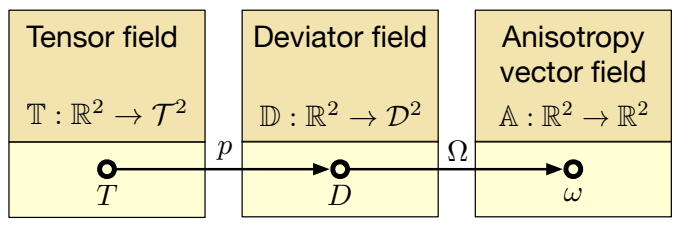

Figure 4: Map a tensor field to an anisotropy vector field via a projection $p$ and an isometry $\Omega$ between a deviator field and an anisotropy vector field.

Let $\mathbf{e}=(\cos \theta, \sin \theta) \in \mathbb{R}^{2}$ be a normalized major eigenvector (with an arbitrary choice of orientation) of tensor $T .{ }^{\dagger}$ Let $A:=\lambda_{1}-$ $\lambda_{2}$, where $\lambda_{1}$ and $\lambda_{2}$ are the eigenvalues associated with the tensor $T$ whose corresponding deviator is $D$. The isometric mapping $\Omega$ : $\mathscr{D}^{2} \rightarrow \mathbb{R}^{2}$ maps an element $D \in \mathscr{D}^{2}$ to

$\omega:=\Omega(D)= \begin{cases}A(\cos 2 \theta, \sin 2 \theta) & \text { if } A \neq 0 \\ (0,0) & \text { if } A=0, \text { for degenerate pts. }\end{cases}$

By construction, the set of degenerate points of $\mathbb{T}$ is the same as the set of critical points of its corresponding anisotropy vector field $\mathbb{A}$, and is given by $(\Omega \circ p)^{-1}(0)$. Please note, however, that the global topological structure including the separatrices of an anisotropy vector fields is not the same as that of its corresponding tensor field. The connectivity among the critical points and the degenerate points largely differs, as shown in Fig. 5.

Well Group Theory. The definition of robustness for vector or tensor fields builds upon the well group theory [CPS12]. Given a mapping $f: \mathbb{X} \rightarrow \mathbb{Y}$ with $\mathbb{X}$ and $\mathbb{Y}$ as topological spaces and $A \subseteq \mathbb{Y}$, well group theory studies the stability, or robustness, of the homology of

$\dagger$ Alternatively, e could be represented by a complex number $\mathbf{e}=$ $\exp (i \theta)=\cos \theta+i \sin \theta \in \mathbb{C}$, where $\theta$ is the complex argument or phase. Then $\mathbf{e}^{2}=\cos 2 \theta+i \sin 2 \theta$. The mapping $\Omega$ uses the Cartesian coordinates of $\mathbf{e}^{2}$ and scales it by $A$. 

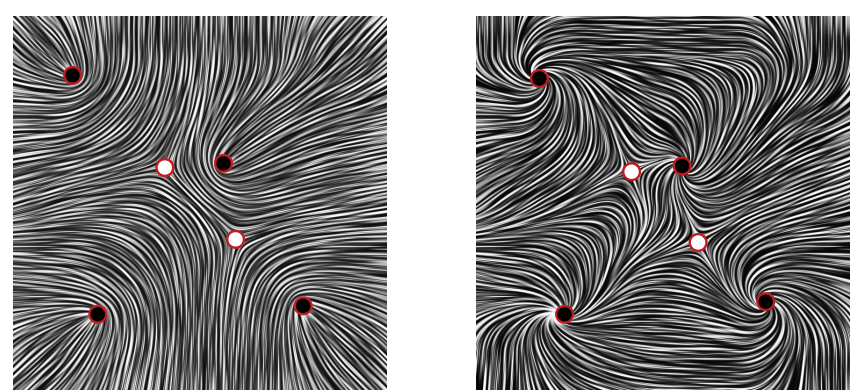

Figure 5: An analytically defined tensor field (left) and its corresponding anisotropy vector field (right) displayed using a texture visualization. The degenerate points from the tensor field on the left correspond to the critical points from the vector field on the right. A trisector (white dot) on the left is transformed into a saddle point on the right. A wedge point (black dot) on the left becomes a source or a sink on the right.

the pre-image of a set $A, f^{-1}(A)$, with respect to perturbations of the mapping $f$. The homology of a space, in a nutshell, measures its topological features such as connected components, tunnels, and voids. In particular, the 0-dimensional homology describes the connected components of the space; and its rank captures the number of connected components. For details, we refer to the original disposition of well group theory [EMP10, EMP11, CPS12].

In the case of a $2 \mathrm{D}$ vector field, the set $A$ of interest is the set of critical points (i.e., zeros). The mapping $f$ is given as $f: \mathbb{R}^{2} \rightarrow \mathbb{R}^{2}$, and one studies the stability of the critical points $f^{-1}(0)$ with respect to a perturbation of the field under the $L_{2}$ norm. For a detailed explanation of the definition of robustness for vector field, see [WRS*13].

In the case of a $2 \mathrm{D}$ symmetric tensor field $\mathbb{T}: \mathbb{R}^{2} \rightarrow \mathscr{T}^{2}$, we study the robustness of its degenerate points by mapping $\mathbb{T}$ to its corresponding anisotropy vector field $f:=\mathbb{A}: \mathbb{R}^{2} \rightarrow \mathbb{R}^{2}$. Let $f_{0}: \mathbb{R}^{2} \rightarrow \mathbb{R}$ be the tensor anisotropy (or equivalently, the maganitute) defined as the difference of the eigenvalues $f_{0}(x)=\|f(x)\|_{2}=$ $\left|\lambda_{1}-\lambda_{2}\right|$, for all $x \in \mathbb{R}^{2}$. Let $\mathbb{F}_{r}$ denote the sublevel set of $f_{0}$, $\mathbb{F}_{r}=f_{0}^{-1}(-\infty, r]$, that is, all points in the domain with an anisotropy smaller than $r$. A value $r>0$ is a regular value of $f_{0}$ if $\mathbb{F}_{r}$ is a manifold (possibly with boundary) and $\forall$ sufficiently small $\varepsilon>0$, $f^{-1}[r-\varepsilon, r+\varepsilon]$ retracts to $f^{-1}(r)$, otherwise it is critical [CPS12]. In particular, $\mathbb{F}_{0}$ is the set of degenerate points, as shown in Fig. 6 and Fig. 7. Let $\mathbb{T}^{\prime}: \mathbb{R}^{2} \rightarrow \mathscr{T}^{2}$ be another tensor field, whose corresponding anisotropy vector field $h$ is an $r$-perturbation of $f$, as shown in Fig. 7). Then $h^{-1}(0)$ is a subspace of $\mathbb{F}_{r}$, that is, we have an inclusion $h^{-1}(0) \subseteq \mathbb{F}_{r}$. This is the most important property that allows us to apply well group theory results in a simple setting. The relation $h^{-1}(0) \subseteq \mathbb{F}_{r}$ induces a linear map $j_{h}$ from the 0 -dimensional homology group of $h^{-1}(0)$ to the 0-dimensional homology group of $\mathbb{F}_{r}$. The well group considers all possible $r$ perturbations $h$ of $f$ and is defined as

$$
\mathrm{U}(r)=\bigcap_{h} \mathrm{i} m j_{h} .
$$

Assuming a finite number of critical points, the rank of $U(0)$ is the number of critical points of $f$. The rank of the well group decreases monotonically as $r$ increases. Establishing a relation to degree theory suggests an algorithm to compute the rank of the well groups in a simple way [CPS12].

If $r$ is a regular value of $f_{0}$, then the rank of the well group $\mathrm{U}(r)$ is the number of connected components $C \subseteq \mathbb{F}_{r}$ such that its degree $\mathrm{d} e g(C) \neq 0$.
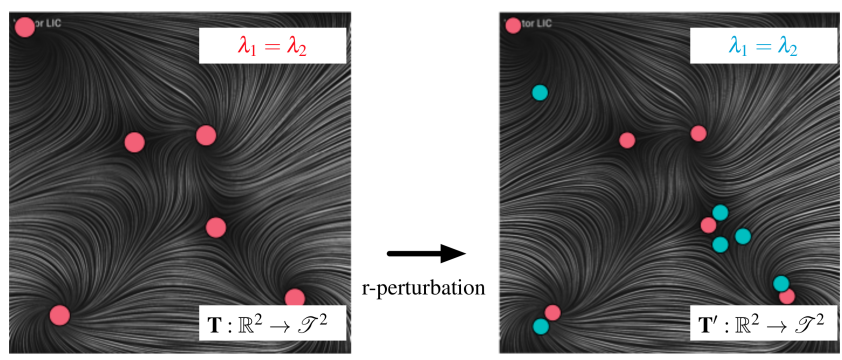

Figure 6: Left: A texture representation of the major eigenvector field of a tensor field $\mathbb{T}$ overlaid with red dots marking the degenerate points of $\mathbb{T}$. Right: Perturbing the field $\mathbb{T}$ has resulted in a tensor field $\mathbb{T}^{\prime}$ whose degenerate points in blue are overlaid with the texture of $\mathbb{T}$.

The well diagram keeps track of the changes of the rank of the well group when increasing the perturbation $r$. Values $r$ where the rank of the well group drops by $k$ are the nodes of the diagram. They are assigned a multiplicity $k$ [CPS12]. Each point in the well diagram is a measure of how resistant a homology class of $f^{-1}(0)$ is against perturbations of the mapping $f$ [EMP11].
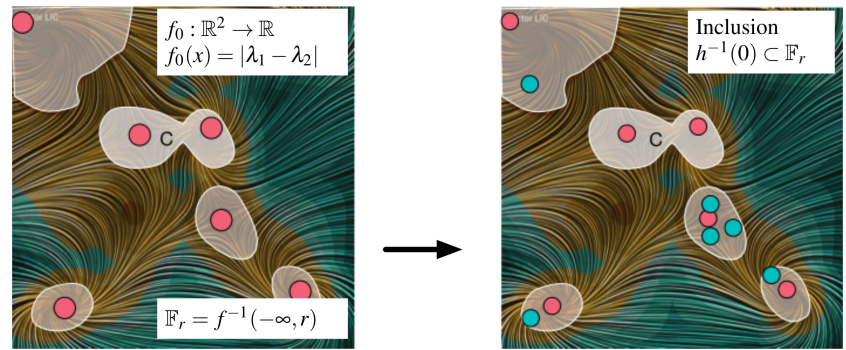

Figure 7: Left: $\mathbb{F}_{r}$ denotes the sublevel sets of the tensor anisotropy. The set $\mathbb{F}_{0}$ is the set of all degenerate points of the field. Right: Given a r-perturbation $h$, then the set of degenerate points of $h$ is a subset of the levelset $\mathbb{F}_{r}$.

Robustness of Degenerate Points. In the setting of 2D tensor fields, the robustness of a degenerate point $x_{i}$ can be described by the robustness of its corresponding class in $U(0)$ that it generates. The robustness of degenerate points in $f$ can be computed by constructing an augmented merge tree of $f_{0}$ that keeps track of the connected components of $\mathbb{F}_{r}$ augmented with their degree. The robustness of a degenerate point is then the height of its lowest degree zero ancestor in the merge tree; see Section 4.3 for algorithmic details surrounding the augmented merge tree.

Transforming a tensor field $\mathbb{T}$ to its corresponding anisotropy 
vector field $f:=\mathbb{A}$ greatly simplifies the extension of robustness from vector fields to the setting of the tensor fields. First, via the projection $p$, an $r$-perturbation in $f$ corresponds to an $r$ perturbation in $\mathbb{D}$. Second, via the mapping $\Omega \circ p$, the degenerate points of $\mathbb{T}$ map to the critical points of $f$.

Therefore, the robustness of degenerate points $x$ for a tensor field $\mathbb{T}$ would resemble the robustness of its corresponding critical point for the anisotropy vector field $f$. Therefore, highly robust degenerate points in a tensor field enjoy nice theoretical properties the same way as their counterparts in the vector field setting. We describe these properties here for completeness, whose proofs can be found in [WH17].

Let $f: \mathbb{R}^{2} \rightarrow \mathbb{R}^{2}$ be an anisotropy vector field that arises from a tensor field $\mathbb{T}$. An $r$-perturbation $\mathbb{T}^{\prime}$ of $\mathbb{T}$ gives rise to an $r$ perturbation $h$ of $f$. We have the following lemmas.

Lemma 3.1 (Nonzero Degree Component for Tensor Field Perturbation) Let $r$ be a regular value of $f_{0}$ and $C$ a connected component of $\mathbb{F}_{r}$ such that $\operatorname{deg}(C) \neq 0$. Then for any $\delta$-perturbation $h$ of $f$, where $\delta<r$, the sum of the degrees of the critical points in $h^{-1}(0) \cap C$ is $\operatorname{deg}(C)$.

Lemma 3.2 (Zero Degree Component for Tensor Field Perturbation) Let $r$ be a regular value of $f_{0}$ and $C$ a connected component of $\mathbb{F}_{r}$ such that $\operatorname{deg}(C)=0$. Then there exists an $r$-perturbation $h$ of $f$ such that $h$ has no degenerate points in $C, h^{-1}(0) \cap C=\emptyset$. In addition, $h$ equals $f$ except possibly within the interior of $C$.

Since the degenerate points of $\mathbb{T}$ map bijectively to the critical points of $f, f$ has no critical points in a path-connected region $C \subset \mathbb{R}^{2}$ if and only if $\mathbb{T}$ has no degenerate points in $C$. Intuitively, Lemma 3.1 implies that for a path-connected component $C$ of $\mathbb{F}_{r}$ with nonzero degree, any $\delta$-perturbation (where $\delta<r$ ) preserves the degree of $C$. That is, if $C$ contains any degenerate point with a robustness greater than $r$, then any $\delta$-perturbation can not cancel it. Lemma 3.2 implies that if a degenerate point contained in a zero degree path-connected component $C$ of $\mathbb{F}_{r}$ has a robustness less than $r$, then there exists an $r$-perturbation that cancels such a degenerate point.

\section{Computing Robustness for Degenerate Points}

Robustness for degenerate points quantifies their structural stability and can be used as an importance measure for the hierarchical simplification of tensor fields. Robustness for critical points has been applied successfully in the setting of vector field simplification [SWCR15]. Its theoretical foundation for the analytic tensor field simplification has been established and is summarized in Section 3 . In the following section, we will transition from the continuous setting to the discrete setting where the tensor field is defined on a triangulation. Using triangulations is common for much of the work based on combinatorial methods. One can use linear interpolations for data defined on triangulations, which simplify the computations. It is possible that new pairs of degenerate points may be generated due to interpolations, however those will have very low robustness values and will disappear early in the simplification, see e.g. Fig. 11.

In addition, the criteria that are used for the extraction of critical points for triangulations can be easily extended to quad meshes, for instance, using the rotation angles along edges for the extraction of degenerate points. The computation of the merge tree for quad meshes would require an analysis of all cases that could appear for a bi-linear interpolation, which is fairly straightforward. For the rest of this paper, we focus on data defined on triangulations.

Since the major and minor eigenvector fields have dual topology and the same degenerate points, we restrict our discussion to the major eigenvector field; eigenvectors from now on always refer to the major eigenvectors.

\subsection{From Analytic to Discrete Setting}

We now consider a tensor field $\mathbb{T}$ restricted to a triangulation $K$ of the domain, a subset of $\mathbb{R}^{2}$. Let $v_{i}=\left(x_{i_{1}}, x_{i_{2}}\right) \in \mathbb{R}^{2}$ (for $i=1 \ldots n$ ) denote the vertices and $e_{i j}$ the oriented edges in $K$. A discrete tensor field $\mathbb{T}$ is a mapping that assigns to each position $v_{i} \in K$ a symmetric second-order tensor $\mathbb{T}\left(v_{i}\right)=T_{i}$.

For a proper application of the robustness framework, it is required that an $r$-perturbation of the discrete field implies an $r$ perturbation of the interpolated field with respect to the Frobenius norm. A component-wise, piece-wise linear (PL) interpolated field fulfills this criterion. It changes continuously when the discrete tensor field changes at the vertices, as shown in Fig. 8 (a). Thus the amount of perturbation for the interpolated field is bounded above by the amount of perturbation at the vertices.

In comparison, a feature-based interpolation does not guarantee such a continuous dependency; it separates the direction interpolation from the eigenvalue interpolation, where small variations in the input data can result in large variations of the interpolated results. Such an interpolation relies on an explicit direction assignment of tensors in neighboring vertices, which can switch discontinuously, as shown in Fig. 8 (b). Although the feature-based interpolation has been used in some applications, it is not suitable for the robustness framework presented here.

\subsection{Stable Extraction of Degenerate Cells}

While the computation of degenerate points in 2D tensor fields is not numerically challenging, it is possible to generate results that are not consistent with the input data, e.g., degenerate points on edges may be detected twice in both adjacent cells or not at all. For this reason, we have decided to use a combinatorial algorithm that guarantees a topologically consistent result.

Given a discretization of the domain, computing the robustness of a degenerate point does not require its accurate spatial location within a cell. Therefore, it is sufficient to find the cells containing degenerate points, referred to as the degenerate cells. We propose an edge-labeling approach that detects degenerate cells based on the winding number of the enclosed degenerate points. The algorithm is simple and uses only the two deviator components. No computation of eigenvectors and eigenvalues is required. This makes our approach fundamentally different from the algorithm of Nair et al. [SNAHH11]. The work in [SNAHH11] also uses edge labels, however they are based on the eigenvector field 


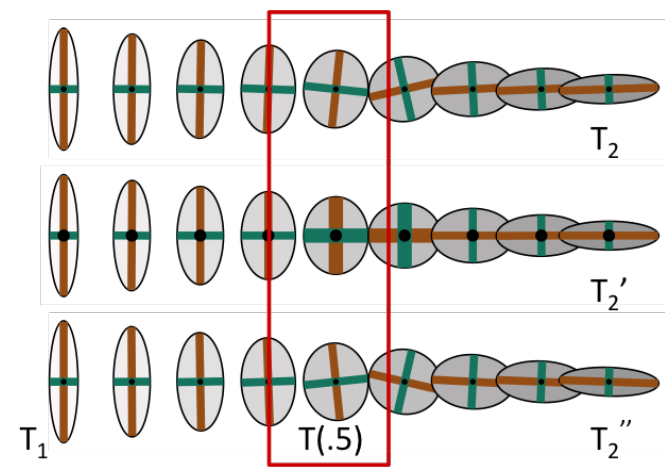

(a) Component-wise interpolation

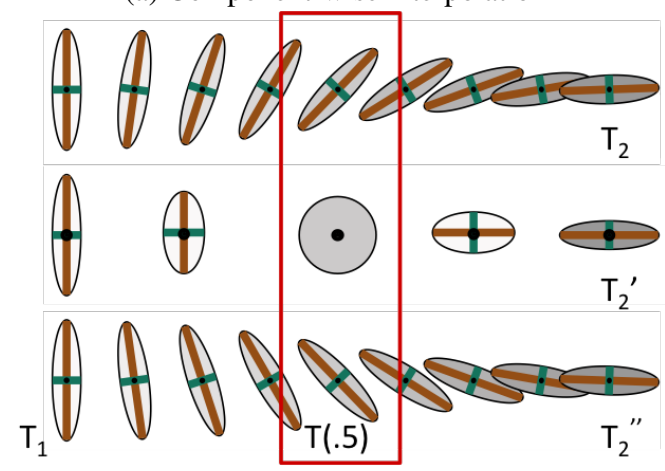

(b) Feature-based interpolation

Figure 8: Interpolation schemes between two tensors $T_{1}$ and $T_{2}$. (a) Component-wise interpolation. (b) Feature-based interpolation, which decouples directions from eigenvalues. Note that the component-wise interpolated field changes continuously at T(.5) when one of the tensor changes from $T_{2}$ to $T_{2}^{\prime}$ and to $T_{2}^{\prime \prime}$, respectively; whereas the feature-based interpolated field introduces discontinuities.

\begin{tabular}{|c|c|c|}
\hline $\begin{array}{c}\text { Discrete } \\
\text { tensor field } \\
T_{i}\end{array}$ & $\rightarrow$ & $\begin{array}{c}\text { Edge labels } \\
l_{i j}\end{array}$ \\
\cline { 1 - 1 } $\begin{array}{c}\mathbf{0} \\
\text { Vertices } v_{i}\end{array}$ & $\begin{array}{c}\mathbf{0} \\
\text { Edges } e_{i j}\end{array}$ \\
$\begin{array}{c}\text { Eegenerate } \\
\text { cell detection } \\
\text { \& classification }\end{array}$ \\
$\begin{array}{c}\mathbf{0} \\
\text { Triangles } t_{i j k}\end{array}$ \\
\hline
\end{tabular}

Figure 9: The detection and classification of degenerate cells is based on edge labels encoding the rotation direction of the eigenvectors. The labels are computed only once to ensure a consistent detection of degenerate cells.

computation; they only allow the detection of critical points and do not provide a direct classification of the degenerate points.

Algorithm Overview. Given a discrete tensor field $\mathbb{T}$ defined on a triangulation, the algorithm consists of the following major steps, summarized in Fig. 9: (i) computing the label for each edge $e_{i j}$ (as \pm 1 ), which keeps track of rotation direction of the eigenvector along $e_{i j}$, and (ii) detecting and classifying cells (e.g., triangles) $t_{i j k}$ that contain degenerate points based on the edge labels. Step (i) contains all numerical computations, whereas step (ii) is purely combinatorial to guarantee a consistent extraction.

Algorithm Assumptions. The algorithm builds upon the following assumptions: (a) by construction, we use PL interpolation of the tensor components; (b) no degenerate tensors lie on the edges or vertices; and (c) the eigenvector directions of tensors on neighboring vertices are always different. To guarantee (b) and (c), we apply a symbolic perturbation of the tensor field described below.

Algorithm Foundation. Let $e_{i j}$ be an oriented edge of the triangulation pointing from vertex $v_{i}$ to vertex $v_{j}$. We assume that the edges of the triangle have a consistent counterclockwise orientation. Let $D_{i}:=\left[\begin{array}{cc}\Delta_{i} & F_{i} \\ F_{i} & -\Delta_{i}\end{array}\right]$ be the deviators of the tensors $T_{i}$. By inspecting the eigenvectors of the interpolated field when moving from vertex $v_{i}$ to $v_{j}$ along the edge $e_{i j}$, we notice the following properties based on simple computations: (i) the absolute value of the rotation angle $\alpha_{i j}$ of the eigenvectors is limited by $\pi / 2$, and (ii) the direction of the rotation, the sign of $\alpha_{i j}$, is given by the sign of $F_{j} \Delta_{i}-F_{i} \Delta_{j}$, see Appendix. The direction is undetermined if this expression is equal to zero; that is, if and only if the rotation angle $\alpha_{i j}$ is either 0 or $\pm \pi / 2$. In general the rotation angle lies in the interval $\alpha_{i j} \in[-\pi / 2, \pi / 2]$. When $\alpha_{i j}=0$, the eigenvectors coincide; and when $\alpha_{i j}= \pm \pi / 2$, there is a degenerate point on the edge. Both cases are excluded according to algorithm assumptions (b) and (c).

Consider now a triangle $t_{i j k}$ with edges $e_{i j}, e_{j k}$ and $e_{k i}$. We know that $\alpha_{i j}+\alpha_{j k}+\alpha_{k i}= \pm n \pi$, where $n \in \mathbb{N}$ is the winding number of the triangle. Furthermore, we know that for any two edges of the triangle, $\left|\alpha_{i j}+\alpha_{j k}\right|<\pi$, which leads to the following observations:

- For degenerate triangles, $n \neq 0$, the rotation direction is the same on all edges and the maximum value for $|n|$ is 1 .

- For non-degenerate triangles, $n=0$, the triangles always contain positive and negative edges.

- There is at most one first-order degenerate point in each cell.

Edge Labels. Based on these properties, we define the edge labels, which are used to detect and classify degenerate triangles. The edge label $l_{i j}$ of an oriented edge $e_{i j}$ is defined as

$$
l_{i j}=\operatorname{sgn}\left(F_{j} \Delta_{i}-F_{i} \Delta_{j}\right)=-l_{j i}
$$

The edge label takes values \pm 1 . According to assumptions (b) and (c), the value 0 is excluded.

Triangle Classification. Let $t_{i j k}$ be a triangle with edges $e_{i j}, e_{j k}$ and $e_{k i}$ in a clockwise order. Then,

$$
t_{i j k} \text { is } \begin{cases}\text { degenerate (wedge point) } & \text { if } l_{i j}=l_{j k}=l_{k i}=1 ; \\ \text { degenerate (trisector) } & \text { if } l_{i j}=l_{j k}=l_{k i}=-1 ; \\ \text { non-degenerate, } & \text { otherwise. }\end{cases}
$$

Virtual Perturbation. In the following sections, we assume that the data is given as a deviator field. To guarantee assumptions (b) and (c), we propose the following perturbation of the field by changing the value $F$ or $\Delta$ to $F+\varepsilon$ and $\Delta+\varepsilon$, respectively, with a small real value $\varepsilon>0$ below the machine precision. In practice, we introduce a tag for each component of the deviator, keeping track 


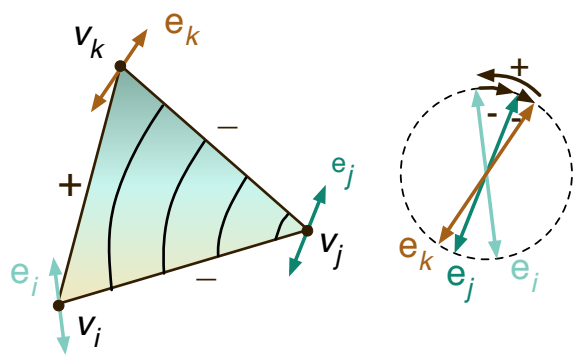

(a)

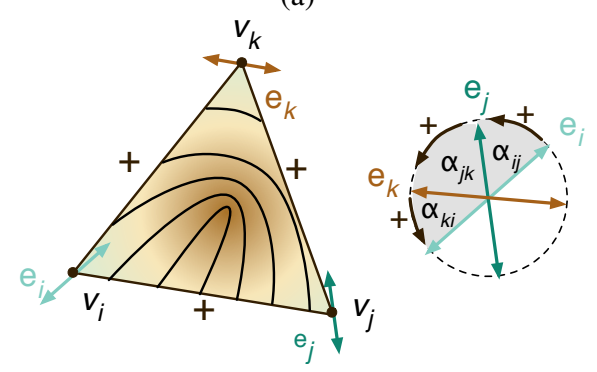

(b)

Figure 10: Direction encoding edge labels allows simple detection and classification of degenerate cells. (a) For a triangle without a degenerate point, the rotation directions along the edges differ, resulting in different edge labels for the edges. (b) For a degenerate triangle containing a wedge point, the rotation along all edges is the same and thus all edge labels have the same sign.

of its perturbation. All tensors are initialized with a zero tag. First, we do a sweep through the data, eliminating degenerate tensors on the vertices. Second, we again sweep through all vertices $v_{i}$, from small to large indices, considering the labels for all outgoing edges $e_{i j}$ (where $j>i$ ). If $l_{i j}=F_{j} \Delta_{i}-F_{i} \Delta_{j}=0$, we perturb the tensor with the higher index $j$. If $\Delta_{i} \neq 0$, we perturb $F_{j}$. If $\Delta_{i}=0$, we perturb $\Delta_{j}$. An already-perturbed tensor is used to determine the label. In some cases, a value may be perturbed more than once.

\subsection{Robustness Computation}

Given a tensor field and a classification of its degenerate cells, we now construct a hierarchy of degenerate points based on their robustness. Specifically, given an input tensor field, we generate its corresponding anisotropy vector field, construct an augmented merge tree, and compute the robustness of its critical points. The ranking of the robustness values implies a multi-scale hierarchy of the critical points, which in turn, allows the exploration of the same hierarchy among their corresponding degenerate points in the original tensor field.

The most commonly used algorithm for merge tree computation assumes a piecewise linear (PL) interpolation of the scalar field inside each cell. This assumption has the advantage that all critical points, namely local maxima, local minima and saddle points, are restricted to the cell vertices. A standard algorithm is proposed by Carr et al [CSA00] based on vertex sorting. Most of the recent parallel implementations also make a PL assumption [MW13].
We now review, on a high-level, the computation of a merge tree for a function defined on a triangulated domain, adapting the description in [MW13]. A merge tree keeps track of the evolution of components in the sub level sets of a scalar function and summarizes their global connectivity [MW13]. Assume our input is a triangulation (simplicial complex) $K$ together with a scalar function $f$ defined on its vertices $V, f: V \rightarrow \mathbb{R}$. In our setting, $f$ is the magnitude of an anisotropy vector field converted from a tensor field. We extend the function $f$ to the underlying space $|K|$ (that is, the union of its simplicies) using PL interpolation and obtain a function $f:|K| \rightarrow \mathbb{R}$.

By definition, merge trees track the connectivity of the sub level sets $K_{a}=f^{-1}(-\infty, a]$. We consider two points $x, y \in|K|$ to be equivalent, denoted as $x \sim y$, if they belong to the same connected component of the sub level set and they have the same function value. Formally, the merge tree is the quotient space $|K| / \sim$, which glues together points in $|K|$ that are equivalent under the relation $\sim$ [MW13]. To understand the definition of a merge tree on the triangulated domain, we increase the function value $a$ from $-\infty$ to $\infty$ and track the creation and merging of branches in the tree. At the local minima of $f$, we create a new branch. As $a$ increases, branches are extended and merged at saddle points. The global maximum of $f$ becomes the root of the tree.

We follow the standard approach in computing merge trees [CSA00], which maintains a disjoint-set data structure. For PL function, only the 1-skeleton of $K$ matters to the computation of the merge tree [MW13]. The algorithm initialize each vertex as its own set and process them in order of increasing function values. The key to the algorithm is to keep track of the lower neighbors $v$ of the current vertex $u$ : "if the sets containing $u$ and $v$ are different, the highest-valued representative in the set of $v$ is linked to $u$ in the merge tree; the two sets are united." [MW13] The running time is determined by the sorting of the vertices and the union-find algorithm. The running time for the standard approach is $O(n \log n+m \alpha(n))$ where $n$ is the number of vertices, $m$ is the number of edges in the triangulation, and $\alpha(n)$ is the inverse Ackermann function.

For robustness computation, we further augment the merge tree with degree information at each node of the merge tree. That is, we record an integer to each node that is the degree of the corresponding component in the sub level set. Recall the robustness of a critical point (degenerate point) is therefore the height of its lowest degree zero ancestor in the merge tree.

However, in the case of an anisotropy vector field, the PL assumption is violated in many cells in the triangulation. In our case the scalar field of interest is the anisotropy field which is given as $A=\left|\lambda_{1}-\lambda_{2}\right|=\sqrt{1 / 4 \operatorname{tr}^{2}(T)-\operatorname{det}(T)}$ for a tensor $T$ at a point. This field is not linear for linearly interpolated tensor components but exhibits a quadratic behavior. Local minima are frequently located inside a triangle, and saddle points are located on the edges. An example is shown in Fig. 11(a), where a wedge and a trisector in two neighboring triangles both have a very low robustness value. Assuming a linear interpolation of the anisotropy field inside the triangles would result in an incorrect, high robustness value for both degenerate points. Therefore, using PL interpolation on the original triangulation leads to incorrect robustness values. 


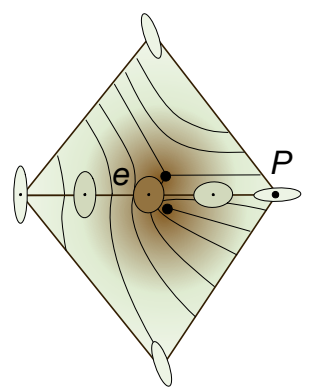

(a)

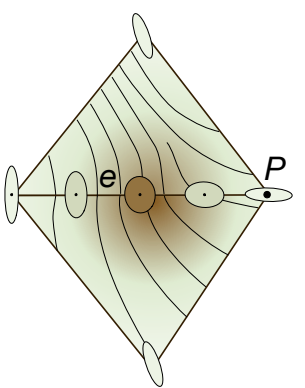

(b)

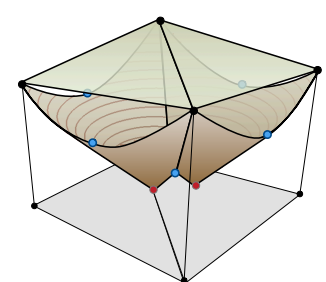

(c)

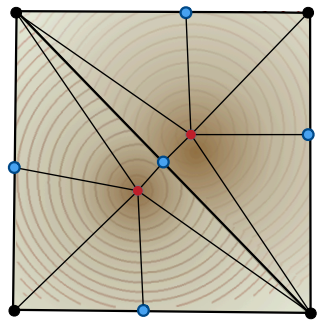

(d)

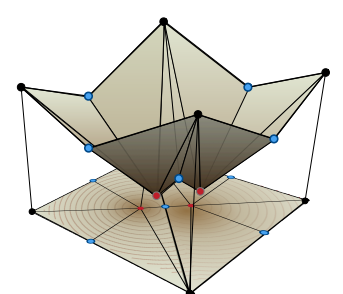

(e)

Figure 11: (a) Two adjacent triangles, each containing a degenerate point. The field is defined by liner interpolation of tensor components for the tensors given in the vertices. The robustness of the degenerate points is low due to a low anisotropy value on the edge e. (b) Applying a small perturbation to the tensor at vertex $P$, the degenerate points cancel each other and disappear. (c) The quadratic behavior of the anisotropy in the two adjacent cells in (a) is shown as colored height field (brown means low and turquoise means high value, respectively). The light green triangles on top show the linearly interpolated anisotropy in these triangles. However, in this case, the computation leads to incorrect, high robustness for the degenerate points. (d) When applying a subdivision of the triangles around local minima, the piecewise linear anisotropy interpolation results in correct robustness values in (e). (e) The triangles on top demonstrate the behavior of the linearly interpolated anisotropy.

This problem can be solved by inserting additional vertices in all local minima (of the anisotropy field) inside a triangle and on the triangle edges, as illustrated in Fig. 11(d)-(e). There are at most four additional vertices per cell, leading to six triangles adjacent to a local minimum (instead of one). On this subdivided structure, the PL algorithm leads to correct robustness results, however at the cost of increasing the number of cells. As shown in Fig. 11(e), the linearly interpolated anisotropy around each local minimum based on the subdivision leads to low robustness values that are consistent with the expected values from Fig. 11(a).

\section{Results}

For the purpose of evaluation, we apply our framework to three data sets. The first data set is an analytic one that contains a collection of isolated degenerate points; it allows us to develop an intuition about the resulting degenerate point pairings and their robustness values. The second data set is a slice of a numerical simulation of a stress tensor field from mechanical engineering; it is well understood and is used to verify the correctness of the results. The last and the most complex data set is a slice of a diffusion tensor imaging (DTI) from the human brain that contains more than a thousand degenerate points.

To demonstrate our results, the tensor fields are visualized as texture aligned with the major eigenvector direction, whose color encodes the anisotropy ranging from zero (dark brown) to its maximum value (turquoise). The degenerate cells are highlighted by colored triangles, black for the wedge type and white for the trisector type. The robustness values are superimposed using colored spheres: dark brown for the minimum values, turquoise for the maximum values, and red for $\infty$ (cells that are never canceled).

\subsection{Collection of Isolated Degenerate Points}

We start with a simple analytic data set for a first evaluation of our framework. The data set is constructed by assembling templates of basic linear structures defined by tensors on the vertices of a quad mesh. Using a simple interface, these tensors can be easily manipulated. The eigenvector directions on the vertices of each cell in the quad mesh determine the cell type (trisector, wedge, or regular). The eigenvalues assigned to each vertex can be used to manipulate the anisotropy field and thus the robustness values of the degenerate cells.

Two examples of such tensor fields, Variant I and Variant II, are shown in Fig. 12(a) and (c), respectively. Both fields have the same eigenvectors associated with the generating tensors, except for two tensors in the center of the mesh (in blue for Variant II). The tensor fields are visualized using a texture visualization in Fig. 12(e) and (f), and the anisotropy is shown for Variant I as well. Six degenerate cells are detected for both fields; they coincide and match well with the texture degeneracies. However, the order of cancellation based on robustness among the degenerate points differs, as expected, for both variants. The order of cancellation for each variant is captured in the merge tree, as shown in Fig. 12(b) and (d), respectively. For each variant, two pairs of distinct degenerate cells (in red) with index 1/2 survive the cancellation. The robustness values for the degenerate points are shown using a color coding: brown for the pair of degenerate points that is canceled first, turquoise for the pair that is canceled second, and red for the degenerate points that survive to the end. We also highlight the contours of the anisotropy fields related to the cancellations.

\subsection{Two Point Load}

The second example is the result of a numerical simulation of a stress tensor field by applying two forces to a solid block, as shown in Fig. 13(a). We analyze the topology of a 2D slice parallel to the top surface of the block. The results are shown in Fig. 13(b)-(e). In our context, we always use a texture highlighting the direction of the major eigenvector field of the stress tensor field. This texture helps to highlight the structures surrounding the wedges and the tri- 
Variant I

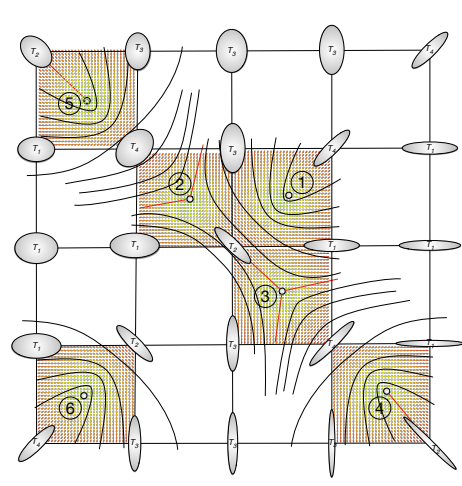

(a)

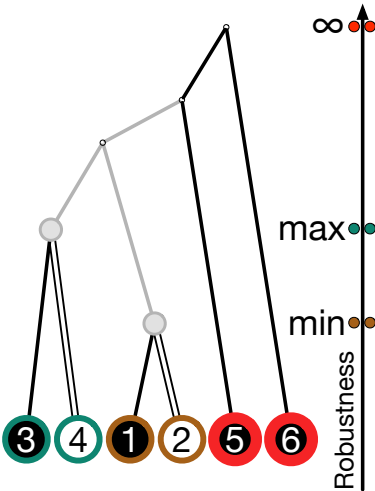

(b)

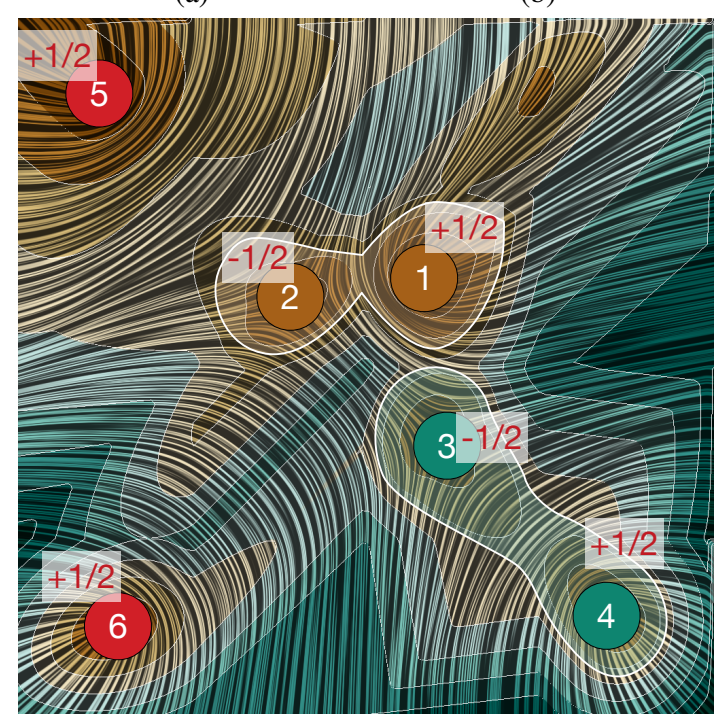

(e)
Variant II

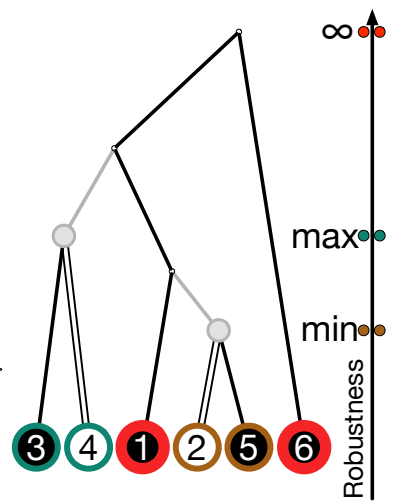

(d) (c)

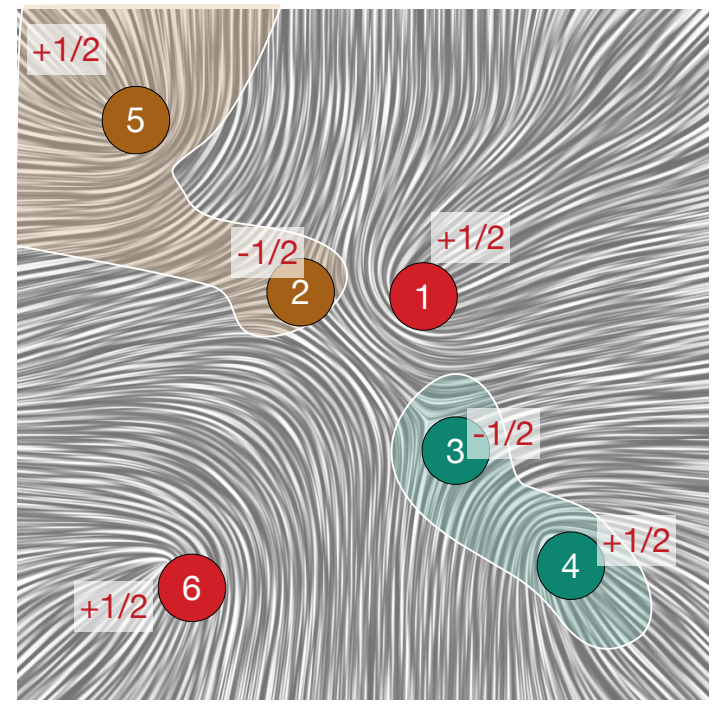

(f)

Figure 12: (a) and (c): Two tensor fields, Variant I/II, are generated as a composition of six isolated degenerate points. The two fields differ only in the anisotropy of two generating tensors, as shown in blue in (c). The two tensor fields are visualized with texture in (e) and $(f)$. For each field, the contours related to the cancellation of two degenerate points are highlighted. The anisotropy is shown for Variant I.

sectors. The extracted degenerate cells are overlaid onto the texture. Note that the degenerate points on the edges are always pushed into a neighboring triangle due to the symbolic perturbation. The robustness values of the degenerate cells are encoded using a brown-toturquoise color map. The degenerate cells that cannot be canceled are highlighted in red.

Our results are consistent with the expected outcome of our framework in Fig. 1(b). Three degenerate cells (corresponding to degenerate points 1, 7, and 21) are never canceled in Fig. 13(b-c), all containing trisector points whose winding number sum up to 1.5 (the same winding number along the boundary of the entire slice). At the second highest simplification level within the hierarchy of the global merge tree, Fig 13(c), five degenerate cells remain: a pair for the upper right region (degenerate points 1 and 7), a pair for the lower left region (degenerate points 19 and 21), and a degenerate cell (degenerate point 20) in the middle representing a separating trisector. The locations of these two pairs of degenerate cells are consistent with the locations of the applied forces. The upper right pair corresponds to a star-like structure, and the bottom left pair corresponds to a circular structure, respectively. Apart from the geometric embedding, our result corresponds precisely to the ideal, hand-drawn sketch in Fig. 1(b).

\subsection{Slice of a Diffusion Tensor Data Set}

For a more challenging data set, we apply our framework to a slice of a DTI data set. We use the entire slice without masking the noisy part outside the brain. To show the data in a 3D context, we embed the slice in a volume rendering of the anisotropy field in Fig. 14(a). Note that, however, this is not an ideal visualization for this data since the resolution of the texture is lower than the frequency of the noise; therefore, artificial structures may be present. All degenerate cells are shown in Fig. 14(c). The data contains more than 1000 degenerate cells, which is too big to draw the merge tree and to make a detailed analysis of each cancellation. Instead, we show 


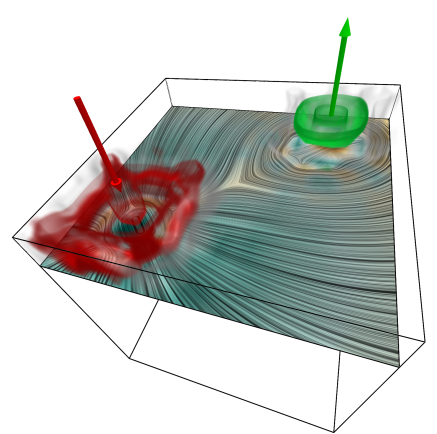

(a)

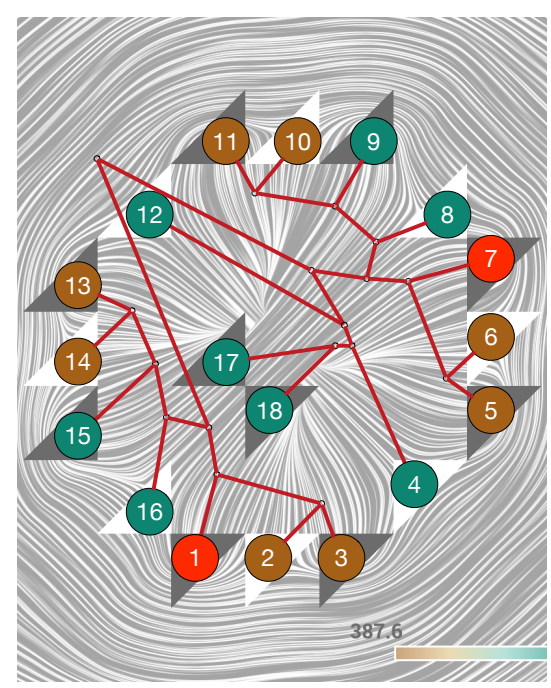

(d)

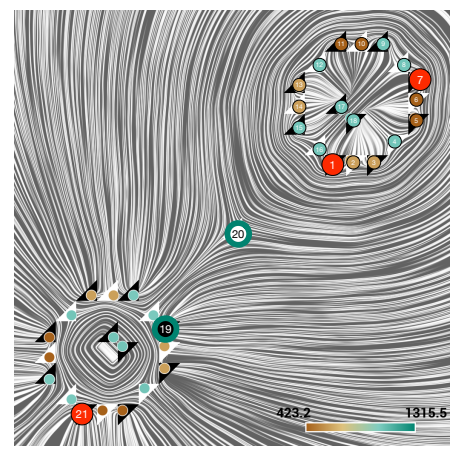

(b)

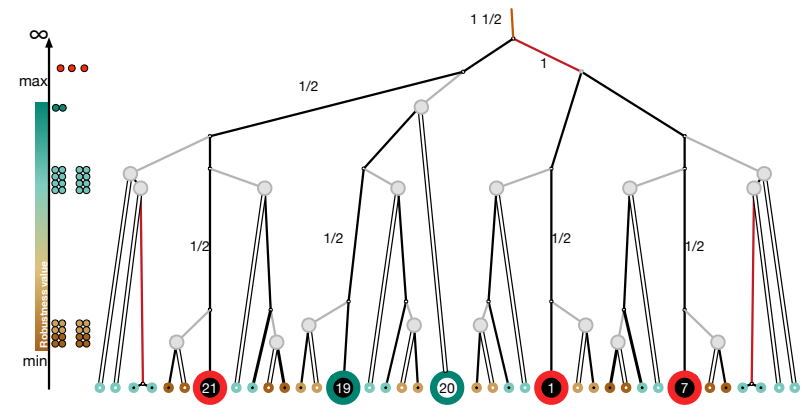

(c)

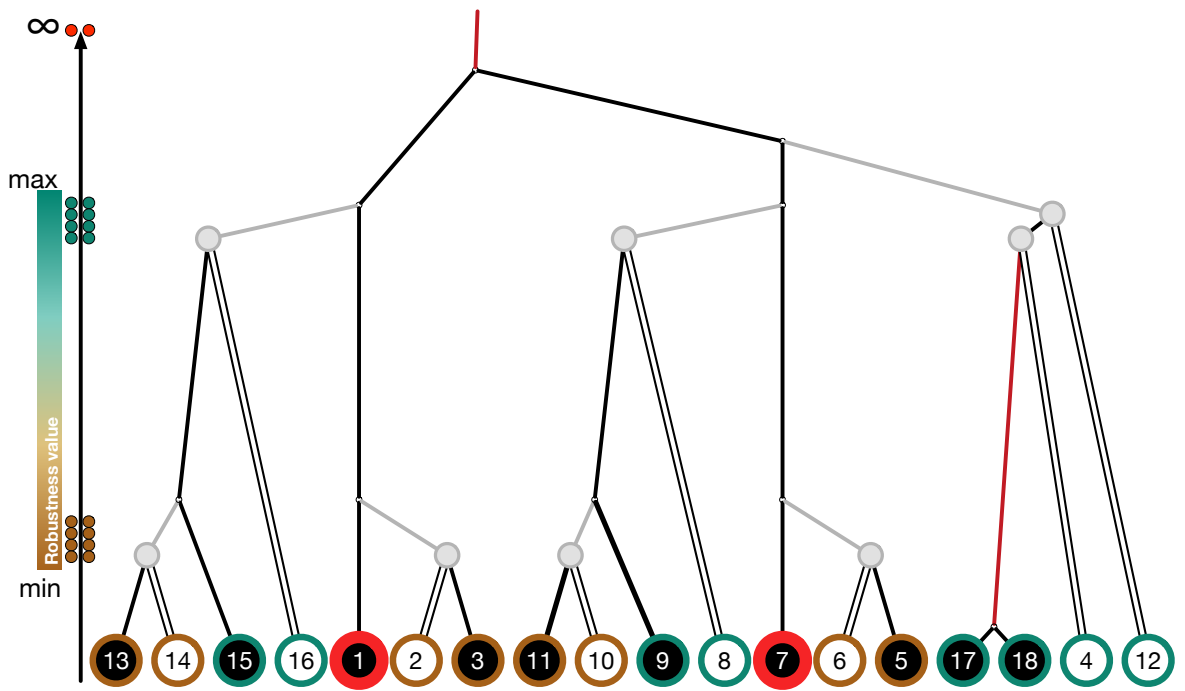

(e)

Figure 13: Visualization of the robustness of a slice of a stress tensor field. (a) A single slice of the data embedded in a 3D context visualized using volume rendering. (b) A textured slice with degenerate cells. White triangles represent trisectors and black ones represent wedges. Degenerate points in each cell are visualized with a brown-to-turquoise colormap. Degenerate points with infinite robustness are in red. (c) The full merge tree. (d) A close-up of the upper right region of $(b)$ with numbered degenerate points. (e) The merge tree encoding the pairwise cancellation of the degenerate points in $(d)$; it is also geometrically embedded in the domain of $(d)$.

a visualization where we filter out the cells with low robustness values, Fig. 14(d). Notice that all the degenerate points with high robustness values are found inside the brain region. A histogram of the robustness values can be seen in Fig. 14(b). A detailed analysis and interpretation of these results are left to future work.

\section{Conclusions}

Combined with previous theoretical results [WH17], our work moves toward a complete, robustness-based implementation of a new tensor field simplification scheme. We generate a hierarchy of degenerate points based on the notion of robustness. By translating a given tensor field into an anisotropy vector field, robustness-based tensor field simplification inherits similar properties from vector field simplification, that is, it enables the pruning of sets of degenerate points according to a quantitative measure of their stability. Besides the actual simplification of the field based on the hierarchy of degenerate points, we discuss some future directions below.
Permutation Metrics. In this paper, we define the perturbation of tensor fields using the infinity norm $L_{\infty}$. It would be interesting to see how an $L_{2}$ norm would lead to different results. We expect the resulting hierarchy to be different for different metrics. We consider the choice of a specific norm as a part of an adaptation to a specific application.

Interpolation Schemes. We currently employ a PL algorithm after subdivision of the triangles such that all local minima of the anisotropy are represented as vertices. This guarantees a correct merge tree computation; however, it is not the most efficient way when dealing with large data. As future work, we will investigate more efficient merge tree implementations that do not need the PL assumption but still give the correct result.

Physical Interpretation. We are also interested in understanding the physical interpretation of the robustness measure. Traditionally, simplifying the curvature tensor field is done implicitly by smoothing the surface. How does this (and other) simplification strategy 


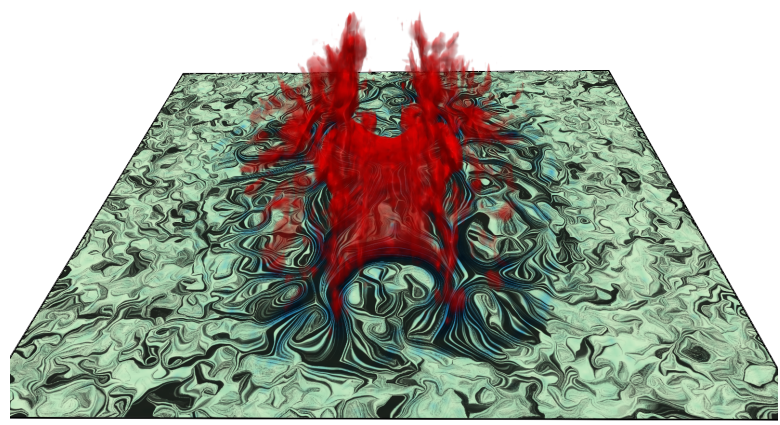

(a)

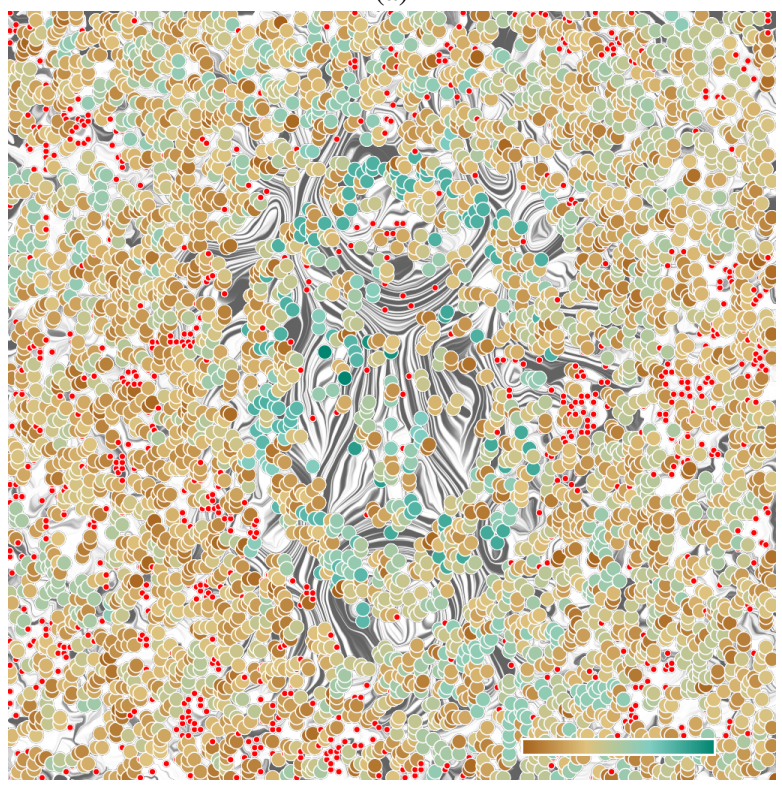

(c)

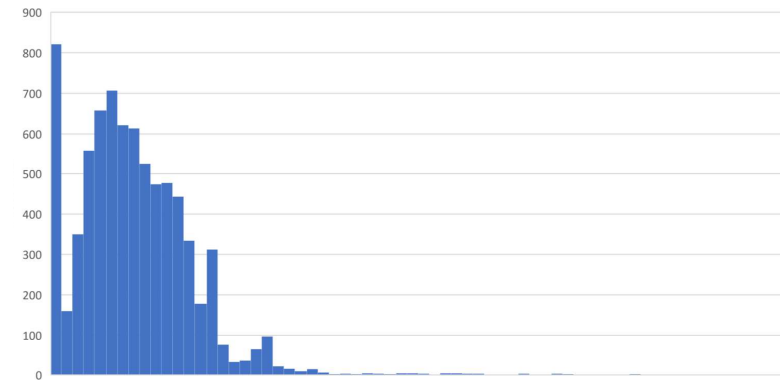

(b)

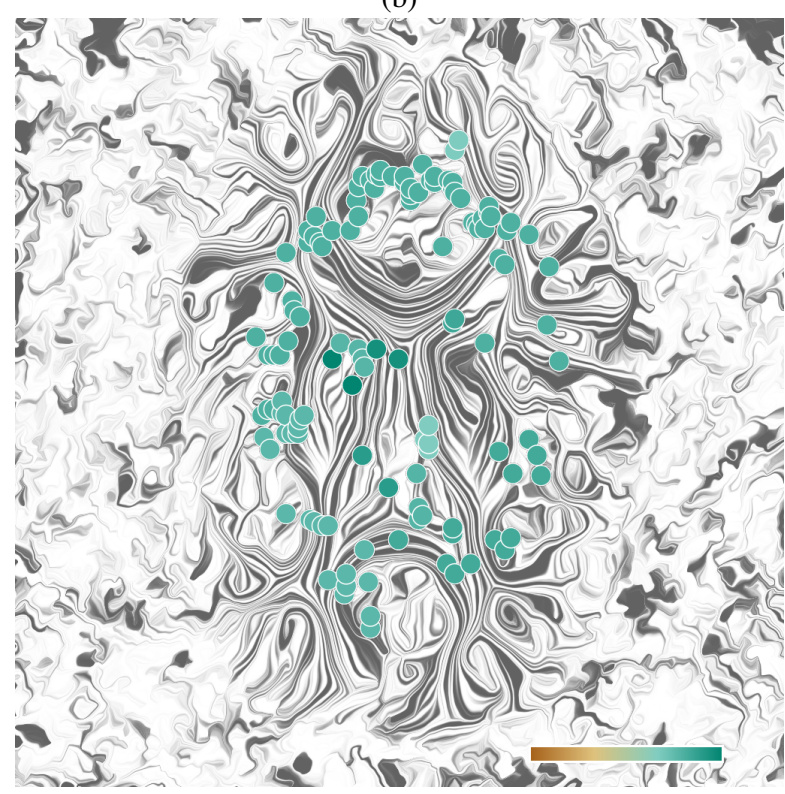

(d)

Figure 14: A slice of a diffusion tensor imaging data set. (a) 3D context visualization using volume rendering of the anisotropy. (b) The histogram of the robustness values of degenerate points. (c) All degenerate cells are color-coded according to their robustness values. (d) Most robust degenerate cells are highlighted in turquoise.

relate to the robustness-based simplification? Alternatively, as we construct simplified fields according to the robustness measure, how does such a simplification correspond to physically meaningful changes to, for instance, a stress tensor field?

Other future directions include a better merge tree construction and robustness computation that handles special cases in Fig. 11; efficient, provably correct implementation in the discrete setting; and the interpretation of the simplified tensor fields in real world applications, such as stress tensor fields and DTI.

\section{Acknowledgement}

This work was supported through the SeRC (Swedish e-Science Research Center) and the ELLIIT environment for strategic research in Sweden. The presented concepts have been realized using the Inviwo open source visualization framework (www.inviwo.org). This work was partially supported by NSF IIS1513616.

\section{References}

[ACSD*03] Alliez P., Cohen-Steiner D., Devillers O., Levy B., Desbrun M.: Anisotropic polygonal remeshing. ACM Transactions on Graphics 22, 3 (2003), 485-493. 1, 2

[ASNZH11] Auer C., Sreevalsan-Nair J., Zobel V., Hotz I.: 2D tensor field segmentation. In Scientific Visualization: Interactions, Features, Metaphors, Hagen H., (Ed.), vol. 2 of Dagstuhl Follow-Ups. Schloss Dagstuhl-Leibniz-Zentrum fuer Informatik, 2011, pp. 17-35. 1

[CPS12] Chazal F., Patel A., Skraba P.: Computing well diagrams for vector fields on $\mathbb{R}^{n}$. Applied Mathematics Letters 25, 11 (2012), 1725-1728. 2, 4, 5

[CSA00] CARR H., SNOEYINK J., AXEN U.: Computing contour trees in all dimensions. Proceedings of the 11th Annual ACM-SIAM Symposium on Discrete Algorithms (2000), 918-926. 8

[De194] Delmarcelle T.: The Visualization of Second-Order Tensor Fields. PhD thesis, Stanford University, 1994. 2, 3

[ELZ02] Edelsbrunner H., Letscher D., Zomorodian A.: Topological persistence and simplification. Discrete \& Computational Geometry 28 (2002), 511-533. 1, 2

[EMP10] Edelsbrunner H., Morozov D., Patel A.: The stability of the apparent contour of an orientable 2-manifold. In Topological 
Methods in Data Analysis and Visualization, Pascucci V., Tricoche X., Hagen H., Tierny J., (Eds.), Mathematics and Visualization. Springer, 2010 , pp. 27-41. 2, 4, 5

[EMP11] Edelsbrunner H., Morozov D., Patel A.: Quantifying transversality by measuring the robustness of intersections. Foundations of Computational Mathematics 11 (2011), 345-361. 2, 4, 5

[KASH13] Kratz A., Auer C., Stommel M., Hotz I.: Visualization and analysis of second-order tensors: Moving beyond the symmetric positive-definite case. Computer Graphics Forum 32, 1 (2013), 49-74. 2

[KSZ*14] Kratz A., SChÖNEICH M., Zobel V., Burgeth B., Scheuermann G., Hotz I., Stommel M.: Tensor visualization driven mechanical component design. IEEE Pacific Visualization Symposium (2014). 1

[MK04] MARINOV M., KobBelt L.: Direct anisotropic quad-dominant remeshing. Pacific Conference on Computer Graphics and Applications (2004), 207-216. 1, 2

[MW13] Morozov D., Weber G. H.: Distributed merge trees. In Proceedings of the 18th ACM SIGPLAN symposium on Principles and practice of parallel programming (2013), ACM. 8

[SNAHH11] SReevalsan-Nair J., Auer C., Hamann B., Hotz I.: Eigenvector-based interpolation and segmentation of 2D tensor fields. In Topological Methods in Data Analysis and Visualization, Pascucci V., Tricoche X., Hagen H., Tierny J., (Eds.), Mathematics and Visualization. Springer, 2011, pp. 139-150. 2, 6

[SRW*16] Skraba P., Rosen P., Wang B., Chen G., Bhatia H., PASCUCCI V.: Critical point cancellation in 3D vector fields: Robustness and discussion. IEEE Transactions on Visualization and Computer Graphics 22, 6 (2016), 1683-1693. 2, 4

[SW14] SKRABA P., WANG B.: Interpreting feature tracking through the lens of robustness. In Topological Methods in Data Analysis and Visualization III, Bremer P.-T., Hotz I., Pascucci V., Peikert R., (Eds.), Mathematics and Visualization. Springer, 2014, pp. 19-37. 4

[SWCR14] Skraba P., WANG B., Chen G., Rosen P.: 2D vector field simplification based on robustness. IEEE Pacific Visualization Symposium (2014). 2

[SWCR15] Skraba P., WANG B., Chen G., Rosen P.: Robustnessbased simplification of $2 \mathrm{D}$ steady and unsteady vector fields. IEEE Transactions on Visualization and Computer Graphics 21, 8 (2015), 930-944. 2, 4, 6

[Tri02] TRICOCHE X.: Vector and Tensor Field Topology Simplification, Tracking and Visualization. $\mathrm{PhD}$ thesis, University of Kaiserslautern, 2002. 2

[TS03] Tricoche X., Scheuermann G.: Topology Simplification of Symmetric, Second Order 2D Tensor Fields. In Geometric Modeling Methods in Scientific Visualization, Hamann B., Müller H., Hagen H., (Eds.). Springer, 2003, pp. 171-184. 2

[TSH01] Tricoche X., Scheuermann G., Hagen H.: Continuous topology simplification of $2 \mathrm{D}$ vector fields. Proceedings of the conference on Visualization (2001), 159-166. 2

[WH17] WANG B., HOTZ I.: Robustness for 2D symmetric tensor field topology. In Modeling Analysis and Visualization of Anisotropy, Hotz I., Özarslan E., Schultz T., (Eds.), Mathematics and Visualization. Springer, 2017. 2, 3, 4, 6, 11

[WRS*13] Wang B., Rosen P., Skraba P., Bhatia H., Pascucci $\mathrm{V}$.: Visualizing robustness of critical points for $2 \mathrm{D}$ time-varying vector fields. Computer Graphics Forum 32, 2 (2013), 221-230. 4, 5

[ZHT07] Zhang E., HAYs J., TURK G.: Interactive Tensor Field Design and Visualization on Surfaces. IEEE Transaction on Visualization and Computer Graphics 13, 1 (2007), 94-107. 2

\section{Appendix: Rotation Direction of Eigenvectors along Edges}

Since the eigenvectors for a deviator and its corresponding tensor are the same, we restrict our considerations to the deviator. The

(c) 2019 The Author(s)

Computer Graphics Forum (c) 2019 The Eurographics Association and John Wiley \& Sons Ltd. interpolated deviator along an edge $e_{i j}$ (with $D_{i}$ and $D_{j}$ defined on its vertices) is given as $D(t)=(1-t) D_{i}+t D_{j}$. We use the following notation

$$
D(t)=\left(\begin{array}{cc}
\Delta(t) & F(t) \\
F(t) & -\Delta(t)
\end{array}\right) .
$$

Assuming there is no degenerate points on the edge, the major eigenvector $v(t)=\left(v_{1}(t), v_{2}(t)\right)=(\cos \alpha(t), \sin \alpha(t))$ is well defined everywhere and changes continuously whereby the angle $\alpha(t)$ is the angle enclosed by $v(t)$ and the $x$-axis. Using $v_{2}^{\prime}=$ $\cos \alpha(t) \cdot \alpha^{\prime}(t), v_{1}^{\prime}=-\sin \alpha(t) \alpha^{\prime}(t)$, and $\sin ^{2} \alpha+\cos ^{2} \alpha=1$ we obtain

$$
\alpha^{\prime}(t)=v_{2}(t) v_{1}^{\prime}(t)-v_{1}(t) v_{2}^{\prime}(t) .
$$

Using the deviator components one obtains the not-normalized eigenvector as $\left(F(t),-\Delta(t)+\sqrt{\Delta^{2}(t)+F^{2}(t)}\right)$. Inserting these vector components into the equation above, we obtain after simplification,

$$
\alpha^{\prime}(t)=\frac{1}{2} \frac{\Delta(t) F^{\prime}(t)-F(t) \Delta^{\prime}(t)}{\Delta^{2}(t)+F^{2}(t)} .
$$

For linearly interpolated components $\Delta^{\prime}=\Delta_{j}-\Delta_{i}$ and $F^{\prime}=F_{j}-F_{i}$ being constants, we finally obtain

$$
\alpha^{\prime}(t)=\frac{1}{2} \frac{F_{2} \Delta_{1}-F_{1} \Delta_{2}}{\Delta^{2}(t)+F^{2}(t)} .
$$

Since the denominator is always greater than zero, the sign and thus the rotational direction is determined by the numerator and is constant along the edge. 